\title{
Epigenetic mechanisms contribute to decrease stearoyl-CoA desaturase 1 expression in the liver of dairy cows after prolonged feeding of high-concentrate diet
}

\author{
T. L. Xu, ${ }^{*} †$ H. M. Seyfert, $†$ and X. Z. Shen ${ }^{* 1}$ \\ ${ }^{*}$ College of Veterinary Medicine, Nanjing Agricultural University, Nanjing, 210095, P.R. China \\ †Leibniz Institute for Farm Animal Biology, Institute for Genome Biology, 18196 Dummerstorf, Germany
}

\begin{abstract}
Subacute ruminal acidosis (SARA) of dairy cattle is a widely occurring but not very overt metabolic disorder thought to impair milk composition. The enzyme stearoyl-CoA desaturase 1 (SCD1) is rate-limiting for the formation of $\Delta-9$ unsaturated fatty acids and thus crucially involved in controlling lipid metabolism in the liver. It is known that SCD1 expression is downregulated during SARA, but the underlying molecular mechanisms are unknown. To study these mechanisms, we enrolled 12 healthy multiparous mid-lactation Holstein cows into a diet-induced SARA experiment. Six cows were fed a high-concentrate diet for 18 weeks (60\% content of high-concentrate to $40 \%$ forage; $\mathrm{HC}$ group), whereas the others received a low-concentrate diet ad libitum (40\% high-concentrate content to $60 \%$ forage; LC group). Sustained low ruminal $\mathrm{pH}$ values ( $\mathrm{pH} 5.6$ maintained for $4 \mathrm{~h} / \mathrm{d}$ ) and reduced milk yield performance $(2.07 \mathrm{~kg} / \mathrm{d}$ less than $\mathrm{LC}$ cows) verified that SARA had been induced in the HC group. Results showed a significantly decreased concentrations of cis9 monounsaturated long-chain fatty acids in plasma collected from hepatic but not portal veins. This was matched by reduced SCD1 mRNA and protein concentrations in HC livers. The expression levels of genes related to lipid formation (DGAT1 and PLIN2) were downregulated during SARA, whereas those of catabolic genes (CPT1A, CPT2, and ACOX1) and some inflammatory genes were upregulated. Expression of $S C D 1$ was downregulated through reduced transcription and abundance of the transcription factor sterol regulatory element-binding protein 1 (SREBP1c).This effect was augmented by local chromatin tightening and DNA methylation at and around the SREBP1c binding site in the $S C D 1$ promoter. Chromatin immunoprecipitation assays confirmed that SARA reduced SREBP1c
\end{abstract}

Received March 16, 2017.

Accepted November 3, 2017.

${ }^{1}$ Corresponding author: xzshen@njau.edu.cn binding at the SCD1 promoter; hence, epigenetic mechanisms are involved in regulating the expression of genes related to long-chain fatty acid modification, partially through downregulation of both SCD1 and SREBP1c in the liver. Our results suggest that in addition to inflammatory genes, SCD1 is also involved in SARA-induced epigenetic regulation and its associated metabolic changes. This knowledge might help to provide a target for intervening against the detrimental metabolic effects of SARA.

Key words: SARA, epigenetic regulation, SREBP1c, chromatin remodeling

\section{INTRODUCTION}

Overfeeding high-yielding dairy cows with high-concentrate diets is a long-standing problem. Feeding highconcentrate diets may provide enough energy to fully exploit cows' genetic potential for milk synthesis, but, in daily practice, there is a risk of eliciting nutritional deficiency diseases, such as SARA (Kleen et al., 2003). Clinically, this disease is characterized by reduced milk synthesis and induction of inflammatory responses (Bauman and Griinari, 2001; Zebeli and Ametaj, 2009; Colman et al., 2010; Loor et al., 2016). High-concentrate diets result in the accumulation of VFA and lactic acid, which decreases ruminal $\mathrm{pH}$ and thus induces acidosis. These perturbations ultimately cause LPS, the major cell-wall component of gram-negative bacteria, to leak from the rumen into the blood (Plaizier et al., 2008; Chen et al., 2012; Chang et al., 2015d; Zhang et al., 2016). The LPS trigger toll-like-receptor 4 (TLR4) signaling, which in turn activates the $N F K B$ complex of transcription factors, thereby throwing a master switch of immune gene regulation (Vaure and Liu, 2014). This initiates the release of proinflammatory cytokines, such as TNFA and IL1 and IL6 (Hayden and Ghosh, 2011). Hence, SARA-mediated systemic circulation of increased LPS levels causes generalized inflammation, affecting both the liver and mammary glands. The liver is the central metabolic organ and maintains the bal- 
ance between lipid utilization and synthesis (Arisqueta et al., 2013). Understanding SARA-mediated alterations of lipid metabolism in this organ is essential for understanding key aspects of SARA pathology.

The enzyme stearoyl-CoA desaturase 1 (SCD1) introduces a cis-9 bond into SFA (Rincon et al., 2012). Its activity is rate-limiting for the formation of $\Delta-9$ UFA. This key step in the formation of triglycerides and cholesterol esters influences milk composition and milk yield in cows (Moioli et al., 2007; Maryam et al., 2016). Moreover, due to the importance of very low density lipoproteins (VLDL), fatty liver occurs when the rate of hepatic triglyceride (TAG) synthesis exceeds the rate of TAG disappearance through either hydrolysis or exportation via VLDL (Pullen et al., 1990; Drackley, 1999). The VLDL metabolism seems to be highly relevant to the expression of SCD1 in the livers of dairy cows. It has also been found that SCD1 activity has a role during inflammation and coping with stress in mice (Chen et al., 2008; Liu et al., 2011). Although some studies have reported decreased SCD1 activity in bovine livers compared with adipose tissues and mammary glands (St John et al., 1991), more recent papers have revealed that transcription of $S C D$ is indeed involved in the hepatic lipid metabolism of lactating cows by supplying extra lipid nutrients (Vahmani et al., 2014). Moreover, $S C D$ expression has also been associated with the effects of sunflower or linseed oil supplementation on lipid metabolism in the livers of goats (Bernard et al., 2009). These studies suggest the liver may contribute to the $\Delta-9$ desaturation of absorbed fatty acid in ruminants.

Sterol regulatory element binding protein-1 (SREBP1) is a key factor controlling the expression of $S C D 1$ and of other genes involved in fat catabolism (Bené et al., 2001; Ren et al., 2016; Xu et al., 2016). It activates the expression of $S C D 1$ and other factors of lipid metabolism by binding to specific sequence motifs called sterol response elements in their promoters (Osborne, 2000). Although knowledge of this mechanism has come from research in nonruminant species, our previous study of the bovine $S C D 1$ promoter has identified a similar stimulatory role of SREBP1c in ruminants (Xu et al., 2016).

However, it is likely that epigenetic mechanisms also contribute to controlling $S C D 1$ expression in a dietdependent fashion. It has been found that feeding mice with either high-carbohydrate or high-fat diets differentially modulated $S C D 1$ expression in livers. This effect was mediated in part by different degrees of $\mathrm{CpG}$ methylation of the SCD1 promoter (Schwenk et al., 2013). Moreover, it has been established in mammary glands of goats and cows that SARA results in a high degree of DNA methylation within crucial CpG islands of the SCD1 promoter, indicating the involvement of epigenetic mechanisms in its regulation (Dong et al., 2014; Tao et al., 2015). In addition, we previously showed that SARA-induced activation of immune genes in the liver is regulated through epigenetic mechanisms (Chang et al., 2015b,c).

The mechanisms for reducing $S C D 1$ expression in the liver during SARA are unknown. The first objective of the current study was therefore to unravel these mechanisms. Second, we wanted to determine how SARA shifts the balance between lipid anabolism and catabolism in the liver. Regarding potential mechanisms for downregulating $S C D 1$ transcription in the liver during SARA, we hypothesized that this may in part be accomplished by reducing SREBP1 binding at the $S C D 1$ promoter, perhaps through compaction of its binding site. We thus built the experimental design of this study on our previously detailed structural and functional characterization of the $S C D 1$ promoter from cattle, including experimental validation that SREBP1 is indeed a pivotal driver of $S C D 1$ expression in the liver.

\section{MATERIALS AND METHODS}

\section{Experimental Design and Sample Collection}

Twelve healthy multiparous mid-lactating Holstein cows $(455 \pm 28 \mathrm{~kg}$ of BW, $31.59 \pm 3.2 \mathrm{~kg} / \mathrm{d}$ of milk yield, and $106 \pm 7$ DIM; mean \pm SD) were fitted with rumen fistula and catheters in the portal and hepatic veins before the trial began. They were randomly divided into 2 groups of 6 cows each to be fed either high-concentrate or low-concentrate diets (HC or LC, respectively). The $\mathrm{HC}$ diet contained $60 \%$ concentrate and $40 \%$ forage and was given as an experimental treatment for $18 \mathrm{wk}$, whereas a diet of $40 \%$ concentrate and $60 \%$ forage served as a control in the LC group. Animals were kept in individual tiestalls and were fed 3 times a day at 0400,1200 , and 2000 h. Dry matter intake was $21.7 \pm 1.1$ per head. Components and nutrient levels of the respective diets were as published in previous studies (Abaker et al., 2017) and are presented in Table 1. Animals were cared for through 4 wk and antibiotics were applied continuously for $3 \mathrm{~d}$ after surgery. Sterilized heparin saline $(500 \mathrm{IU} / \mathrm{mL})$ was used to prevent catheter blockage daily at 8-h intervals until the end of the experiment. Cows were monitored daily for feed ingestion, rectal temperature, and respiratory rate. The experiment began after all animals had made a full recovery from surgery; no cows showed any clinical signs of infection during the experiment. Sample collection and storage methods were as previously described (Abaker et al., 2017). Briefly, blood was sampled $4 \mathrm{~h}$ 
Table 1. The components and nutrients levels of low-concentrate (LC) and high-concentrate $(\mathrm{HC})$ diets

\begin{tabular}{lcc}
\hline Item & LC diet & HC diet \\
\hline Ingredient (\% of DM) & 30 & \\
Corn silage & 30 & 20 \\
Alfalfa & 22.78 & 33.6 \\
Maize & 5.15 & 15 \\
Wheat bran & 9.81 & 9 \\
Soybean meal & 0.92 & 0.53 \\
Calcium phosphate dibasic & 0 & 0.52 \\
Limestone & 0.35 & 0.35 \\
Salt & 1 & 1 \\
Premix & 100 & 100 \\
Total & & \\
Nutritional composition & & \\
NE (MJ/kg) & 6.45 & 6.85 \\
CP (\%) & 16 & 16.2 \\
Ether extract (\%) & 3.96 & 4.15 \\
NDF (\%) & 37.71 & 31.92 \\
ADF (\%) & 22.75 & 17.55 \\
NFC (\%) & 33.43 & 40.31 \\
Ca (\%) & 0.9 & 0.8 \\
P (\%) & 0.45 & 0.45 \\
\hline
\end{tabular}

${ }^{1}$ The premix contained vitamin $\mathrm{A}, 1,900 \mathrm{kU} / \mathrm{kg}$; vitamin $\mathrm{D}, 250 \mathrm{kU} /$ $\mathrm{kg}$; vitamin E, 3,000 mg/kg; niacin, 4,000 mg/kg; Cu, 1,200 mg/kg; Fe, $525 \mathrm{mg} / \mathrm{kg}$; Zn, 13,000 mg/kg; Mn, 5,500 mg/kg; I, 170 mg/kg; Co, $50 \mathrm{mg} / \mathrm{kg} ; \mathrm{Se}, 27 \mathrm{mg} / \mathrm{kg}$.

${ }^{2}$ The calculated nutritional composition values.

after feeding on d 5, 6, and 7 of wk 18. Blood samples were collected through the catheters in the portal and hepatic veins, using 5-mL Vacutainer tubes (supplied by Jiangsu Kangjian Medical Apparatus Co. Ltd., Nanjing, China) containing sodium heparin as an anticoagulant. Plasma was then isolated from the blood samples by centrifugation at $3,000 \times g$ at $4^{\circ} \mathrm{C}$ for 15 min and stored at $-20^{\circ} \mathrm{C}$ before analysis. At wk 18, liver tissue was sampled by punch biopsy after local anesthesia. Samples were immediately snap-frozen in liquid nitrogen and then stored at $-70^{\circ} \mathrm{C}$ as described (Vels et al., 2009).

\section{Ethics Approval}

The experiment was conducted following the guidelines of the Animal Ethics Committee, Nanjing Agricultural University. The Animal Ethics Committee approved the study. Sampling procedures complied with the Animal Research Institute Committee guidelines of Nanjing Agricultural University.

\section{Rumen pH Measurements and Triacylglycerol Content in Liver}

Rumen fluids were sampled through a rumen fistula from ventral sac at 1-h intervals for up to $12 \mathrm{~h}$ on d 5 , 6 , and 7 of wk 18 of the experimental period. $\mathrm{pH}$ was measured immediately using a portable $\mathrm{pH}$ meter (HI 9125; Hanna Instruments, Woonsocket, RI).

\section{Fatty Acid Analysis}

Total lipids were extracted from ruminal fluid and plasma as previously detailed (Folch et al., 1957). Lipids were converted to FAME and then subjected to GC analysis. Long-chain fatty acids were analyzed on CP 7489 columns $(100 \mathrm{~m} \times 0.25 \mathrm{~mm} \times 0.25 \mu \mathrm{m}$; J\&W Advanced Capillary GC Columns, Agilent, Santa Clara, CA) in an Agilent 7890A detector equipped with an autosampler, flame ionization detector, and split injection. Long-chain fatty acids were quantified using known amounts of methyl nonadecanoate as an internal standard. Fatty acid methyl esters were identified by comparing their retention times to those of the standards.

\section{RNA Extraction and Real-Time Quantitative PCR}

Total RNA was isolated from $50 \mu \mathrm{g}$ of liver tissue using TRIzol Reagent (Invitrogen, Carlsbad, CA) according to the manufacturer's instructions (https://tools .thermofisher.com/content/sfs/manuals/trizol_reagent .pdf). One and a half micrograms of total RNA was reverse transcribed (Superscript II, Invitrogen), and the resulting cDNA was purified using a purification kit (Axygen, Tewksbury, MA). The RNA quality was validated as shown in Supplemental Figure S1 (https://doi.org/10.3168/jds.2017-12878). Real-time quantitative (q)PCR was conducted on an ABI 7300 system (Applied Biosystems, Foster City, CA) using gene-specific primer pairs to amplify target cDNA segments with the SYBR Premix EX Taq kit (cat. DRR420A, Takara, Shiga, Japan). Primers (listed in Supplemental Table S1; https://doi.org/10.3168/jds .2017-12878) were designed with Premier 6.0 software (Premier Biosoft International, Palo Alto, CA). Primer efficiencies (Supplemental Table S2; https://doi.org/10 .3168/jds.2017-12878) were evaluated before use. We chose SFRS4 (splicing factor, serine/arginine rich 4) as a housekeeping gene. Its validity as a reference for normalizing gene expression in liver samples has been previously reported (Chang et al., 2015a). The $2^{-\Delta \Delta \mathrm{Ct}}$ method was used for relative quantification (Pfaffl, 2001).

\section{Chromatin Compaction Assay}

Chromatin compaction was analyzed with chromatin accessibility by real-time PCR according to our previous publications, with some modifications (Vanselow 
et al., 2006; Chang et al., 2015b). Briefly, $4 \mu \mathrm{L}$ of suspended nuclei isolated from stored liver tissue were added into a digestion reaction $\left(37^{\circ} \mathrm{C}, 2 \mathrm{~h}\right)$ containing $20 \mathrm{U}$ of $\mathrm{M} s p \mathrm{I}$ for area A and B of the SCD1 promoter (Figure 1). Subsequently, DNA was purified with a purification kit (Axygen) and quantified with a NanoDrop spectrophotometer (Thermo Scientific ND2000USCAN, Wilmington, DE). Similarly treated samples without restriction enzymes were used as DNA input controls. The fraction of uncut target DNA was determined with qPCR assays using $75 \mathrm{ng}$ of DNA from treatment and control reactions using the SYBR Premix EX Taq kit (cat. DRR420A, Takara) and an ABI 7300 system (Applied Biosystems). Primers are listed in Supplemental Table S1 (https://doi.org/103168/jds.2017-12878).

\section{SCD1 Promoter DNA Methylation Assay}

Genomic DNA was isolated with a MiniBEST Universal Genomic DNA Extraction Kit (Cat. No. 9765, Takara, Beijing, China) from liver tissues, purified (purification kit, Axygen, Painted Post, NY), and analyzed by Methyl-Profiler DNA Methylation qPCR Assays, as previously published with some modifications (De Oliveira et al., 2011; de Faria Amormino et al., 2013). This method is based on the fact that DNA digestion with HpaII, but not with its isoschizomer $M s p \mathrm{I}$, is blocked by $\mathrm{C}$-methylation of $\mathrm{CpG}$ dinucleotides. The DNA was predigested with EcoRI to facilitate accurate aliquoting. Subsequently, $2 \mu \mathrm{g}$ of DNA was digested with either $\mathrm{HpaII}$ or $\mathrm{MspI}\left(20 \mathrm{U}, 37^{\circ} \mathrm{C}, 2 \mathrm{~h}\right)$. The DNA input control samples were similarly treated but without adding restriction enzymes. Subsequently, DNA was repurified and requantified. Then, $100 \mathrm{ng}$ was used to quantify uncut DNA in qPCR assays. Primer sequences for amplifying promoter areas A and B are listed in Supplemental Table S1 (https://doi.org/103168/jds .2017-12878). The degree of methylation was calculated as the difference between the ratio of copy numbers obtained after $H p a \mathrm{II}$ or $M s p \mathrm{I}$ digestion, and each relative value is presented as fraction of the undigested DNA input control, set as $100 \%$.

\section{Western Blotting}

Western blot analysis was conducted essentially as described (Xu et al., 2015). Briefly, protein was isolated from liver tissue homogenized in RIPA lysis buffer (Beyotime, Shanghai, China). Equal amounts of protein were separated on $10 \%$ SDS polyacrylamide gels to resolve SREBP1 and SCD1. Proteins were transferred onto nitrocellulose membranes (Millipore, Billerica, MA), which were incubated with primary

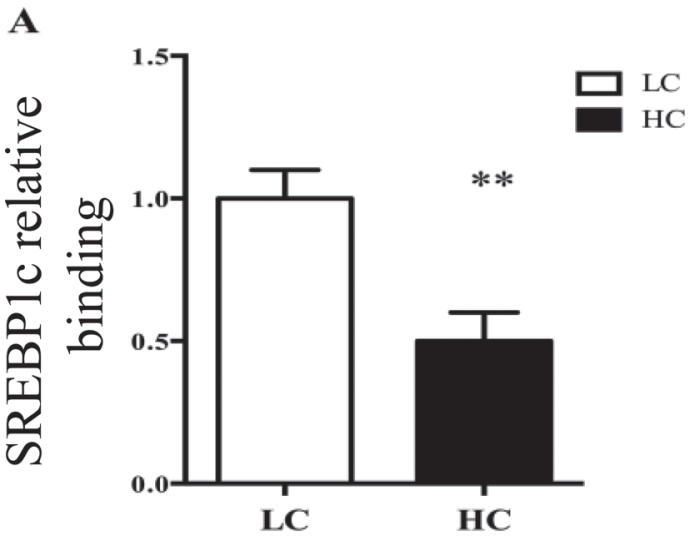

B

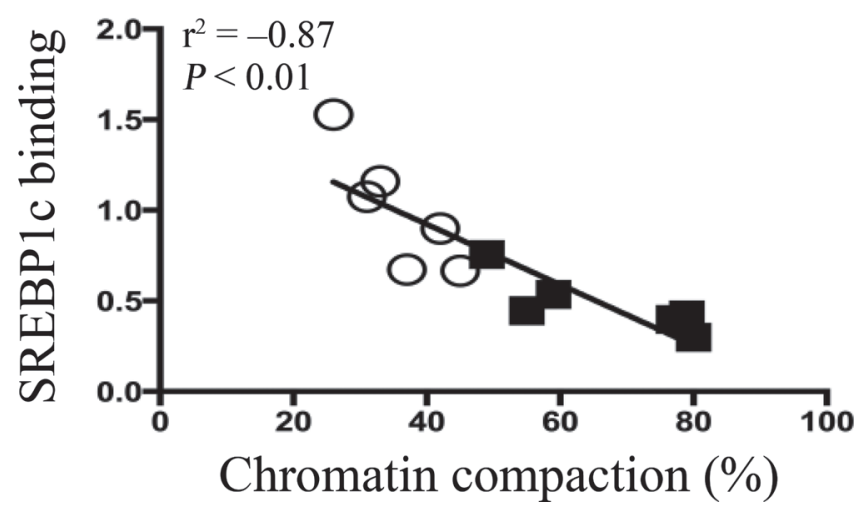

Figure 1. Effect of low-concentrate (LC) and high-concentrate (HC) diets on SREBP1c binding to the SCD1 promoter in the liver. Error bars represent SEM. (A) Level of SREBP1c binding to the SCD1 promoter at area B (cf promoter map in Figure 4) in LC and $\mathrm{HC}$ groups. Six individual samples in each group were involved in generating chromatin immunoprecipitation analysis. ${ }^{* *} P<0.01$, versus LC group. (B) Correlation of SREBP1c binding rate and chromatin protection level at area B.

antibodies against either SCD1 (Goat polyclonal antibody, sc-23016, Santa Cruz Biotechnology, Santa Cruz, CA; diluted to 1:200) or SREBP1 (Mouse monoclonal antibody, ab3259, Abcam, Cambridge, UK; diluted to 1:1,000). After extensive washing, blots were incubated with horseradish peroxidase-coupled secondary antibodies. We used affinity-purified rabbit anti-goat IgG antibodies (E030130-01, Earth Ox, San Francisco, CA; diluted to $1: 10,000)$ to detect SCD1 and rabbit antimouse antibodies (ab6728, Abcam; diluted to 1:5000) to detect SREBP1. Differences in protein transfer efficiency between blots were normalized based on quantification of GAPDH, which was detected with rabbit polyclonal antibodies (AP0066, Bioworld, St. Louis Park, MN; diluted to 1:10,000). Intensities of bands from target genes were quantified with the Quantity One 1-D analysis software (Bio-Rad, Hercules, CA). 
Table 2. The ruminal $\mathrm{pH}$ value from low-concentrate (LC) and high-concentrate (HC) groups

\begin{tabular}{lcccc}
\hline Item $^{1}$ & LC & HC & SEM $^{2}$ & $P$-value \\
\hline Average $\mathrm{pH}$ & 6.02 & $5.90^{*}$ & 0.03 & 0.03 \\
Minimum $\mathrm{pH}$ & 5.65 & $5.50^{*}$ & 0.05 & 0.02 \\
Time $\mathrm{pH}<5.6(\mathrm{~min} / \mathrm{d})$ & 99.00 & $223.00^{*}$ & 30.25 & 0.01 \\
Time $\mathrm{pH}<6.0(\mathrm{~min} / \mathrm{d})$ & 290.00 & 375.00 & 33.86 & 0.25 \\
\hline${ }^{1} \mathrm{pH}$ mean values obtained from 3 continuous days of samples collection. & & \\
${ }^{2} \mathrm{SEM}$ of both LC and HC groups. & & & \\
${ }^{*} P<0.05$, as compared with LC group. & & &
\end{tabular}

\section{Chromatin Immunoprecipitation Assay}

Frozen liver samples were crushed to a powder in a mortar under liquid nitrogen. For each sample, $200 \mu \mathrm{g}$ was homogenized and resuspended in PBS containing protease inhibitor cocktail (Cat. \#11697498001; Roche, Basel, Switzerland) as recommended by the manufacturer. Protein and DNA were cross-linked by adding formaldehyde to a final concentration of $1 \%$. After shaking at room temperature for $10 \mathrm{~min}$, the reaction was stopped with $2.5 \mathrm{M}$ glycine at room temperature. The reaction mix was centrifuged $\left(4^{\circ} \mathrm{C}, 4,000 \times g\right.$ for $5 \mathrm{~min}$ ) and pellets were rinsed with PBS and homogenized in SDS lysis buffer containing protease inhibitors. Crude chromatin preparations were sonicated on ice to yield DNA fragments 200 to $500 \mathrm{bp}$ in length and precleared with salmon sperm DNA-treated protein $\mathrm{G}$ agarose beads $(40 \mu \mathrm{L}, 50 \%$ slurry, sc-2003; Santa Cruz Biotechnology). Precleared chromatin preparations were incubated with $4 \mu \mathrm{g}$ of primary antibodies (Anti-SREBP1, ab3259, Abcam) at $4^{\circ} \mathrm{C}$ for $16 \mathrm{~h}$. As a negative control, 1 sample was incubated with normal rabbit IgG. Protein A/G agarose beads (40 $\mu \mathrm{L}, 50 \%$ slurry, sc-2003; Santa Cruz Biotechnology) was added to capture immunoprecipitated chromatin complexes. Two hundred microliters of precleared chromatin preparation served as input. Finally, DNA fragments were released from the immunoprecipitated complexes by reverse cross-linking at $65^{\circ} \mathrm{C}$ for $1 \mathrm{~h}$ using elution buffer supplemented with 1\% SDS. Quantities of promoter fragments recovered during chromatin immunoprecipitation were determined with qPCR using primers specific for the respective areas of the SCD1 promoter (Supplemental Table S1; https://doi.org/ 103168/jds.2017-12878).

\section{Statistical Analysis}

Data are expressed as the means \pm standard error of the means, and the statistical significance of differences was evaluated with unpaired Student's t-tests. Expression data were log-2 transformed prior analysis, whereas all other data were used as is. For each cow,
$\mathrm{pH}$ data for the 3 last consecutive days of wk 18 were averaged before analysis. Normality was then assessed during statistical analysis and all analyzed variables were found to be normally distributed (Shapiro-Wilk test) using IBM software SPSS 20.0 Statistics for Mac OS (IBM Inc., Armonk, NY). The statistical model of diet treatment ( $\mathrm{LC}$ and $\mathrm{HC}$ ) was set as a fixed effect, whereas cows nested within treatments was used as the random effect. Coefficients of correlation between mRNA expression levels and the degree of chromatin compaction, as well as between the degree of chromatin compaction and percentage of promoter methylation were analyzed using Bivariate Correlations in SPSS 20.0 Statistics. Differences with $P$-values $<0.05$ were considered statistically significant.

\section{RESULTS}

\section{Rumen pH Value Analysis}

The average $\mathrm{pH}$ value in the rumen of $\mathrm{HC}$ cows was lower than that of the LC group $(P<0.05)$. In the $\mathrm{HC}$ group, a $\mathrm{pH}$ value of 5.6 was observed for approximately $4 \mathrm{~h} / \mathrm{d}$ (Table 2). $\mathrm{pH}$ values were measured on 3 consecutive days in wk 18 of treatment and averaged.

\section{Influence of $\Delta-9$ Long-Chain Fatty Acid Content in Plasma Through the Liver}

The contents of UFA cis-9 C14:1, cis-9 C16:1, and cis-9 C18:1 were measured in blood from the portal and hepatic veins (blood influx and efflux from liver, respectively). The HC diet (i.e., SARA) did not alter concentrations of these fatty acids in plasma from the portal vein (Figure 2A); however, they were reduced $(P<0.05)$ in hepatic vein plasma of $\mathrm{HC}$ cows (cis-9 C14:1 and cis-9 C18:1; Figure 2B). The desaturation index, defined as the ratio of cis-9 C18:1/C18:0, was significantly increased in portal vein plasma of $\mathrm{HC}$ animals $(P<0.05$; Figure $2 \mathrm{C})$; however, the concentration of TAG in the liver was not altered in the HC group compared with the LC group (Figure 2E). 


\section{SARA Reprograms Genes Related to Lipid Metabolism and Inflammatory Response}

We used several candidate genes to profile the physiological effects of SARA on the metabolism of fatty acids and insulin in the liver and to assess inflammation. The results are shown in Table 3.

Lipid Metabolism. Expression of SCD1 was 3 fold lower in the livers of the HC group than in the LC control group $(P<0.05)$, validating our model.
LC

$\mathrm{HC}$

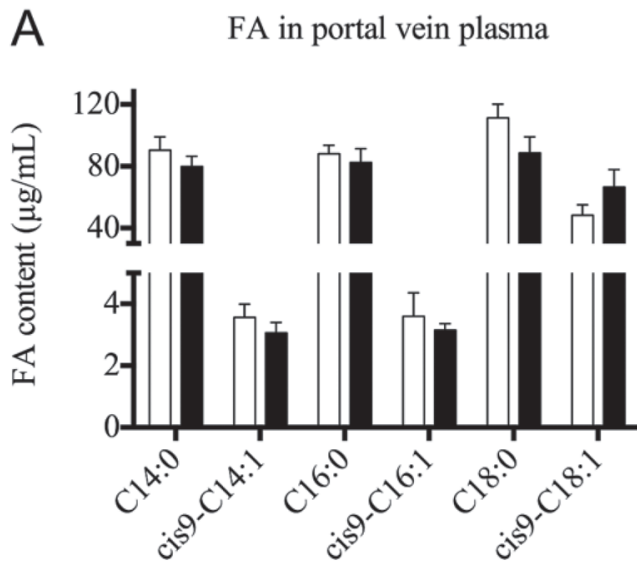

C Desturation index in portal vein
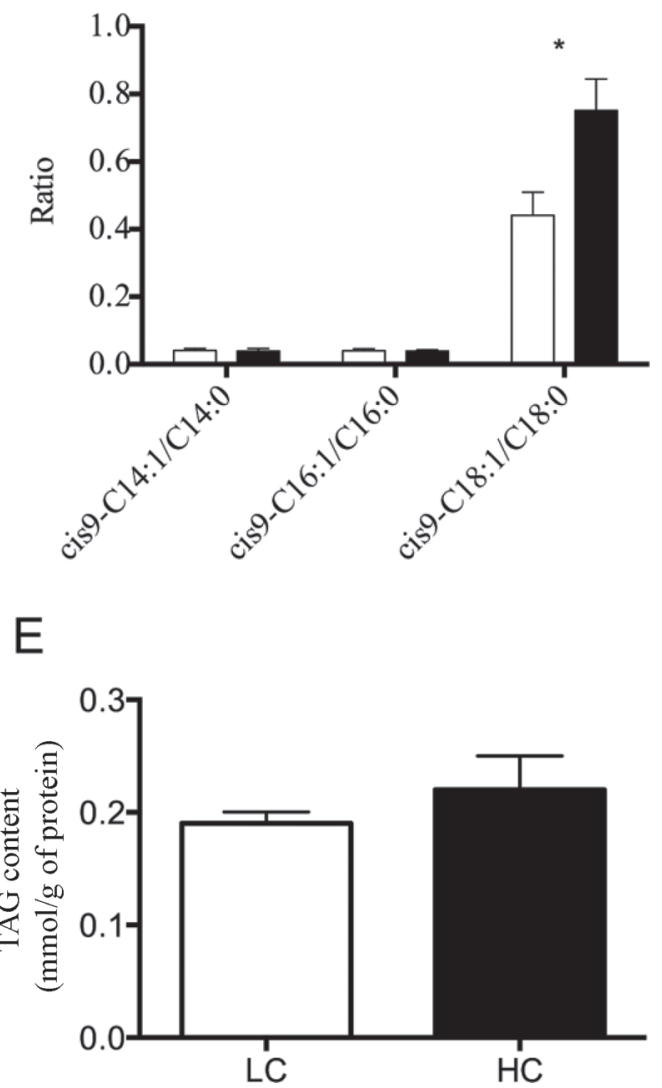

B FA in hepatic vein plasma

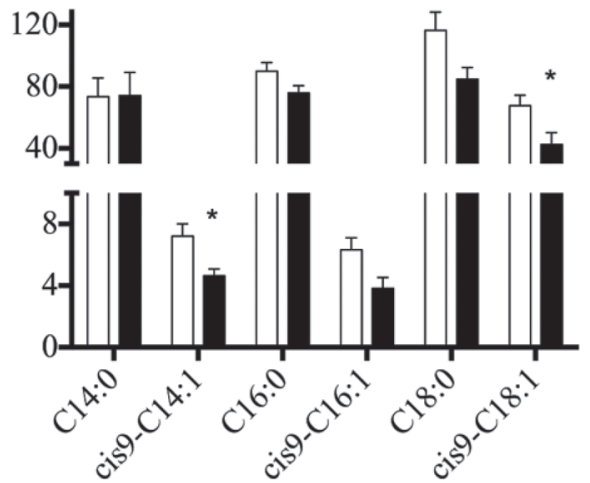

D Desturation index in hepatic vein

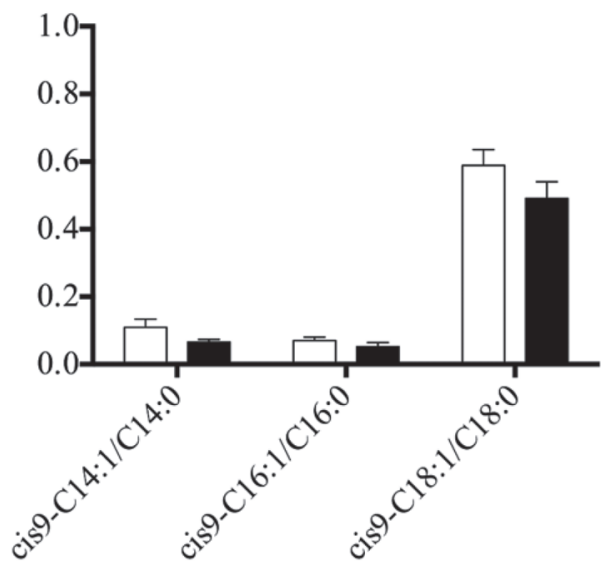

Figure 2. Concentrations of cis-9 MUFA in portal (A) and hepatic (B) vein plasma. Desaturation index was calculated from cis-9 MUFA to their corresponding SFA in portal vein plasma (C) and hepatic vein plasma (D). Triglyceride (TAG) concentration was counted in the liver (E). Values are presented as means $\pm \mathrm{SEM} ;{ }^{*} P<0.05$ versus low-concentrate $(\mathrm{LC})$ group. $\mathrm{HC}=$ high-concentrate group; FA $=$ fatty acid. 
Table 3. Relative mRNA concentration of candidate genes in the liver of cows from the high-concentrate (HC) group

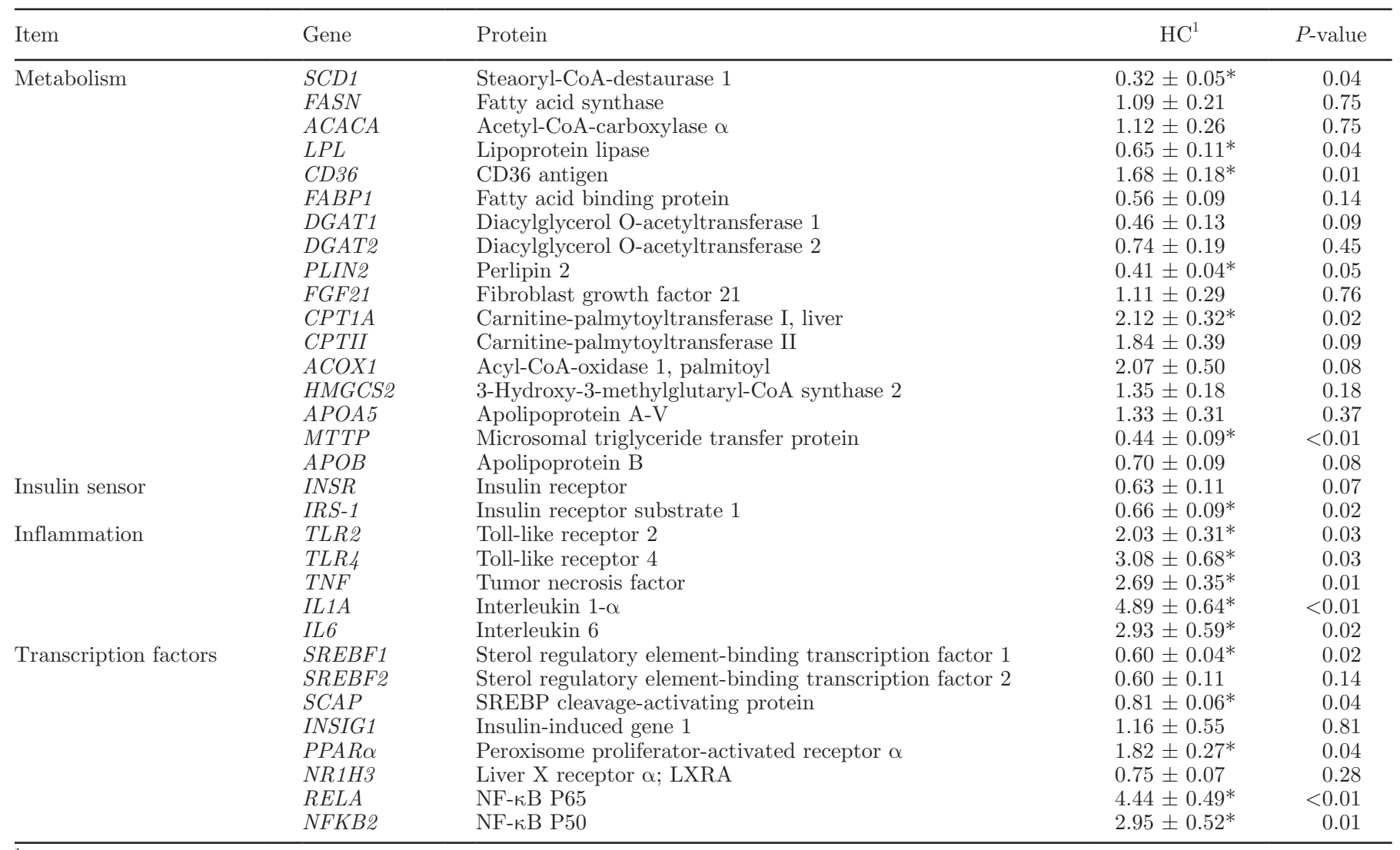

${ }^{1}$ Values are means \pm SEM of the mRNA concentration in livers of $\mathrm{HC}$ cows, relative to the respective mean as measured in low-concentrate (LC) cows, set as 1.0.

*Significant difference from LC group, $P<0.05$.

Otherwise, no diet-related influence was found regarding the expression of fatty acid synthase $(F A S N)$ or acetyl-CoA-carboxylase $\alpha(A C A C A ; P=0.75)$. However, expression of genes related to fatty acid binding was altered, as shown by increased expression of $C D 36$ (also known as fatty acid translocase) and decreased expression of $L P L$ (lipoprotein lipase) in the $\mathrm{HC}$ group $(P<0.05)$. Genes associated with lipid formation such as DGAT1 (triacylglycerol synthesis) and PLIN2 (lipid droplet formation) were downregulated $(P<0.09)$. Genes related to fatty acid oxidation $(C P T 1 A, C P T 2$, and $A C O X 1$ ) were all upregulated approximately 2 fold in the HC group $(P<0.09)$. The genes MTTP and $A P O B$ (lipoprotein metabolism) were both downregulated during SARA in $\mathrm{HC}$ cows $(P<0.08)$.

We used Western blots to determine if SARA also reduces the abundance of the SCD1 enzyme and the SREBP1c transcription factor in cow livers (Figure 3). The abundance of both factors was on average reduced by approximately $40 \%$ in the livers of $\mathrm{HC}$ cows $(P<$ $0.05)$.

Insulin Receptors. The induction of SARA led to a decrease of insulin receptors in the liver, due to down- regulation of IRS1 and INSR compared with control $(P \leq 0.07)$.

Inflammation. Expression levels of 5 inflammationrelated genes (TLR2, TLR4, TNFA, IL1A, and IL6) were highly upregulated 2 to almost 5 fold in the HC group $(P<0.05)$.

Relevant Transcription Factors. Consistently, the expression of the key transcription factor SREBP1c driving the expression of genes being involved in lipid formation was reduced in the $\mathrm{HC}$ group, as was the activation-dependent ligand SCAP $(P<0.05)$. Subacute ruminal acidosis upregulated the expression of PPARA, which is known to stimulate the expression of genes related to fatty acid oxidation and the p65 and p50 transcription factors, which act as nuclear factor$\kappa \mathrm{B}$ subunits $(P<0.05)$.

\section{SARA Induces Chromatin Remodeling of the SCD1 Promoter}

To learn more about the gene regulatory mechanisms triggered in the liver by the SARA condition, we asked whether chromatin remodeling is involved 

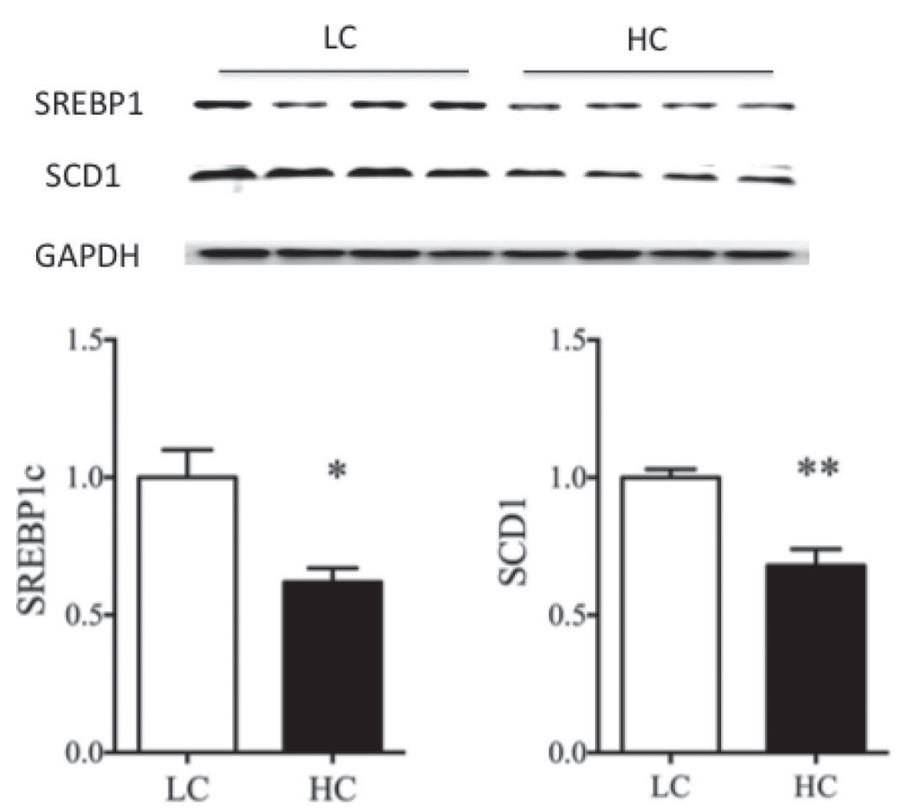

Figure 3. Lipogenic protein expression in the liver of dairy cows fed with low- (LC) or high-concentrate (HC) diet. Relative protein expression of SREBP1c and SCD was normalized with GAPDH. Values are presented as means $\pm \mathrm{SEM}$ in $\mathrm{LC}$ and $\mathrm{HC}$ group. ${ }^{*} P<0.05$, ${ }^{* *} P$ $<0.01$, versus LC group.

in downregulating SCD1 transcription. The degree of chromatin compaction was measured in 2 areas (area A and B, top panel in Figure 4) of the SCD1 promoter. High-concentrate diet-induced SARA led to chromatin compaction in both areas $(75 \%$ in area $\mathrm{A}, 64 \%$ in area B; Table 4) compared with the LC group ( $42 \%$ in area A, $36 \%$ in area B; Table $4 ; P<0.01$ ). Additionally, a robust negative correlation was found between the degree of chromatin compaction and SCD1 mRNA levels $\left(\mathrm{R}^{2}=-0.60, P<0.05\right.$ in area $\mathrm{A} ; \mathrm{R}^{2}=-0.71, P<0.05$ in area B; Figures 1 and $4 \mathrm{~A}$ and $\mathrm{B}$ ). Promoters from SARA-suffering animals (circles in Figure 4) exhibited the tightest chromatin compaction and these samples featured the lowest SCD1 mRNA concentrations.

\section{Promoter Methylation Analysis for SCD1 Promoter Selected Sites}

Based on the SCD1 promoter sequence of cattle, we identified 5 '-CpG motifs embedded in the sequences 5 'CCGG-3' in both promoter areas A and B. These are binding sites for the restriction enzyme isoschizomers $H p a \mathrm{II}$ and $M s p \mathrm{I}$; $M s p \mathrm{I}$ cuts this DNA-sequence motif regardless of methylation, whereas $\mathrm{HpaII}$ does so only if the 3'-C-nucleotide is not methylated. The average degree of methylation at both sites was higher in the HC group (0.41 in area A and 0.55 in area B) than in the $\mathrm{LC}$ group $(0.12$ in area $\mathrm{A}$ and 0.10 in area $\mathrm{B} ; P<$
0.01). All data are shown in Table 4. As expected, a significant positive correlation between the level of DNA methylation and chromatin compaction was observed in both areas $\mathrm{A}\left(\mathrm{R}^{2}=0.74, P<0.01\right)$ and $\mathrm{B}\left(\mathrm{R}^{2}=\right.$ $0.88, P<0.01$ ), as shown in Figure $4 \mathrm{C}$ and D. Particularly striking is the very high level of DNA methylation of area B in cows suffering from SARA.

\section{SARA Decreases SREBP1C Binding to SCD1 Promoter in the Liver}

Chromatin immunoprecipitation was used to examine the degree of SREBP1c factor recruitment onto native chromatin of the $S C D 1$ promoter. Chromatin for immunoprecipitation was extracted from liver tissues of LC and HC cows. An antibody against SREBP1c was used to precipitate chromatin harboring fragments of sheared DNA. The average level of SREBP1c binding to the $S C D 1$ promoter in the HC group was decreased 2 fold $(P<0.01 ;$ Figure $1 \mathrm{~A})$. The percentage of the input DNA specifically precipitated by SREBP1 antibody was significantly higher in LC group $(2.14 \pm 0.36)$ as compared with $\mathrm{HC}$ group $(0.89 \pm 0.08 ; P<0.01)$. The percentage of DNA being nonspecifically precipitated by the control IgG were similar in both HC $(0.40$ $\pm 0.06)$ and $\mathrm{LC}(0.46 \pm 0.06)$ group cows. Moreover, we observed a clear and significant correlation $\left(\mathrm{R}^{2}=\right.$ $-0.87, P<0.01)$ when individual values for SREBP1c abundance at the promoter were plotted against the degree of chromatin compaction (Figure 1B), showing an interdependence of both parameters.

\section{DISCUSSION}

Our previous studies verified that long-term feeding with high-concentrate diets induces the SARA condition in dairy cows and downregulates SCD1 expression in the liver and mammary gland (Tao et al., 2015; Xu et al., 2015). Moreover, induction of SARA has also been reported to negatively influence milk production in the same animals used for the current study in terms of milk yield, percentage of milk fat, and the milk fat yield (Abaker et al., 2017). Therefore, we verified that SCD1 transcription and protein expression are decreased in the liver during SARA. As a key result regarding the regulatory mechanisms of $S C D 1$, we have shown that chromatin remodeling at the $S C D 1$ promoter and downregulation of the transcription factor SREBP1c are crucial factors for downregulating SCD1 expression in the liver of cows suffering from SARA. We also suggest that SARA drives lipid metabolism in the liver toward catabolism rather than anabolism. 


\section{Role of SCD1 for Lipid Metabolism in the Cow Liver}

Generally, SCD1 has been considered to be an enzyme functioning in the generation of $\Delta-9$ fatty acyl$\mathrm{CoA}$ in monogastric animals. However, studies have reported relatively low $S C D$ expression in bovine livers compared with lipogenic tissues, such as MG and adipose, indicating low $S C D$ activity in the livers of ruminants (St John et al., 1991). Despite this, an older study suggested similar levels of desaturase activity in both sheep and rat livers (Payne and Masters, 1971). More recently, $S C D$ gene expression was found to be similar in livers and adipose tissue of goats (Bernard et al., 2009), suggesting that the liver may contribute to the $\Delta-9$ desaturation of absorbed fatty acids in ruminants. Moreover, changes in the expression of $S C D$ have
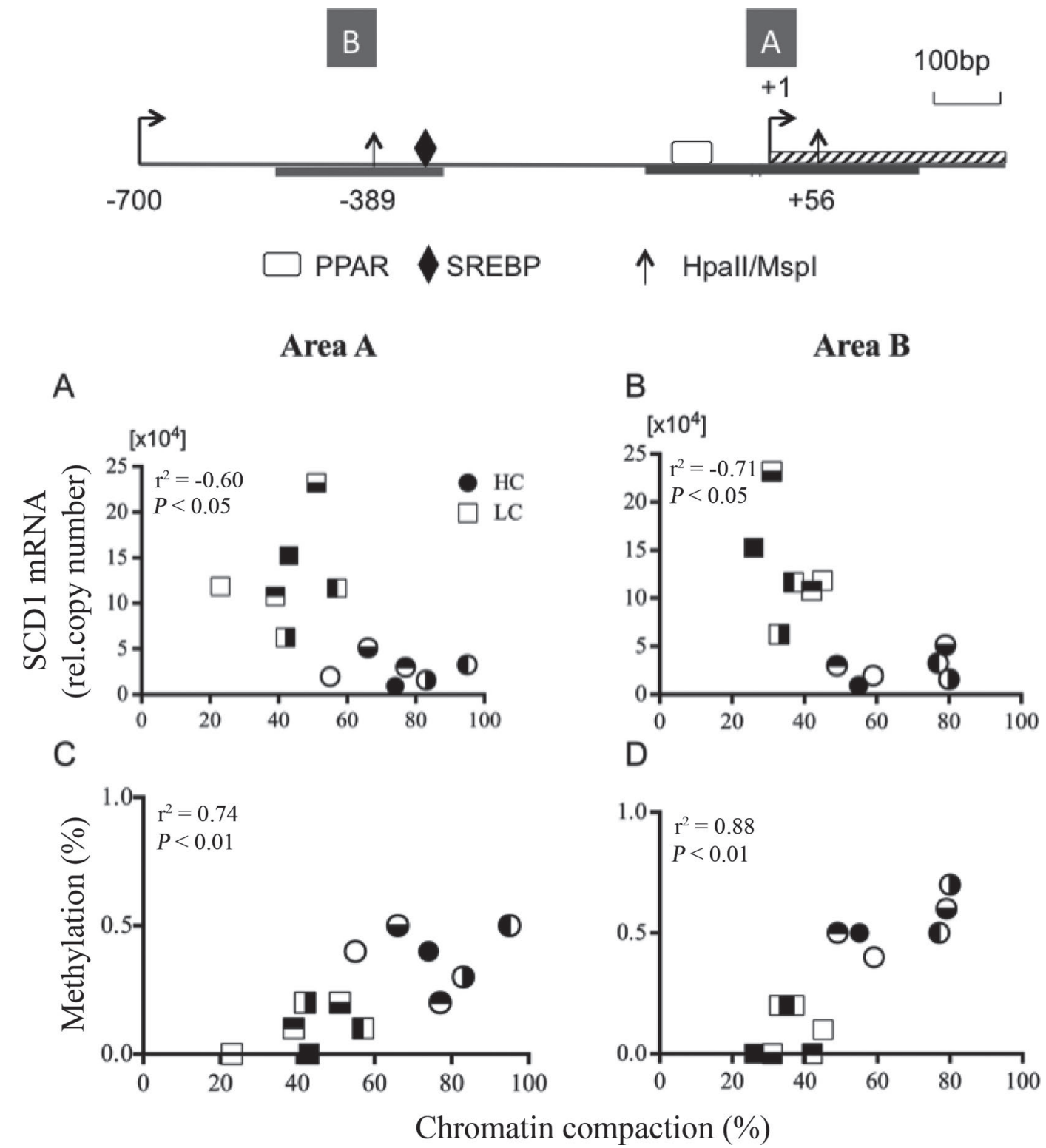

Figure 4. Scatter plot analysis of chromatin compaction, promoter methylation, and mRNA copies in the liver of dairy cows. (Top) Map of SCD1 promoter in cattle, identified by our previous 5' rapid amplification of cDNA ends experiment. Promoter areas A and B are indicated above the graph. Putative transcription factor binding sites are listed below the map, and thick bars indicate the 2 areas (A and B) for chromatin accessibility by real-time PCR and methylation analysis. (A. B) Correlation between $S C D 1$ copy numbers (ordinate) and the degree of chromatin compaction (abscissa, expressed as percentage of undigested control) in area A (A) and B (B) of SCD1 promoter. The lower graphs show the correlation between the degree of chromatin compaction (abscissa, expressed as percentage of undigested control) against the percentages of promoter methylation (ordinate) in area A (C) and B (D). $\square=$ low-concentrate (LC) group; $\bullet$ high-concentrate (HC) group; texture specifies each individual animal. 
been found to be involved in hepatic lipid metabolism of lactating cows by supplying extra lipid nutrients (Vahmani et al., 2014). Thus, understanding the role of SCD1 in bovine hepatic lipid metabolism is still of considerable significance.

\section{Reduced SREBP1 Abundance and Binding to SCD1 Explain Reduced Expression and Abundance of SCD1}

In general, insulin secretion has been suggested to promote SREBP1c expression. However, it has been reported that circulating insulin levels in plasma are higher in cows suffering from SARA (Zebeli et al., 2011; Guo et al., 2013; Xu et al., 2015), including our previous results with the same animals used in the current study (Xu et al., 2015). Insulin resistance occurs when insulin levels are excessively high over a prolonged period of time, reducing the body's sensitivity to the hormone. This may then lead to lower insulin sensitivity and insulin responsiveness in bovine livers (De Koster and Opsomer, 2013). This is in line with the current study, where we have shown that prolonged high-concentrate feeding induces secretion of insulin and subsequently elicits insulin resistance. This idea may be supported by the decreased gene expression of INSR and IRS1 in SARA cows, as the enzymes encoded by these genes play important roles in regulating multiple signaling pathways [i.e., phosphoinositide 3-kinase-serine/threonine kinase/mammalian target of rapamycin (PI3K-Akt/ $\mathbf{m T O R}$ )] in response to insulin in the liver (Boucher et al., 2014). Moreover, we recently found an inhibition of hepatic mTOR signaling in grain-challenged cows (Xu et al., 2017), which has also been suggested to suppress lipid synthesis by inactivating SREBP1 (Düvel et al., 2010). Therefore, elevated insulin levels may fail to raise the expression of SREBP1c due to reduced insulin sensitivity during SARA.
Complementary real-time qPCR and Western blot assays unequivocally showed a reduced abundance of SREBP1c in the livers of cows with SARA. Similarly, complementary assays revealed condensed chromatin at and around the SREBP1c binding site of the SCD1 promoter (area B) together with significantly increased DNA methylation. Thus, SARA-induced chromatin remodeling at this crucial transcription factor binding site undoubtedly hinders binding of the SREBP1c transcription factor, a key driver of bovine $S C D 1$ expression (Xu et al., 2016). Finally, our chromatin immunoprecipitation analysis verified that tighter chromatin compaction correlates with reduced abundance of SREBP1 bound at this SCD1 promoter area in SARA cows. All these lines of evidence together undoubtedly demonstrate in vivo that reduced SREBP1 abundance at its cognate binding site of the $S C D 1$ promoter is a major determinant for downregulating SCD1 expression during SARA in the livers of cattle. Moreover, DNA methylation of that site ensures sustained local chromatin compaction, insulating the promoter against binding of activating transcription factors (Cedar and Bergman, 2009; Smith and Meissner, 2013).

Revealing the involvement of epigenetic mechanisms for downregulating $S C D 1$ expression in the liver aligns with our previous observations that, during SARA, TLR4-mediated signaling activates expression of immune genes in the liver through chromatin decompaction of relevant promoters (Schwenk et al., 2013; Chang et al., 2015d). Accordingly, it has been shown that SCD1 may act as a mediator of inflammatory gene expression by changing DNA methylation levels (Liu et al., 2011; Malodobra-Mazur et al., 2014). However, we previously failed to detect alterations of chromatin compaction at the $S C D 1$ promoter associated with its reduced activity in the livers of cows suffering from acute E. coli mastitis, although we did observe chromatin decompaction of the promoters of immune genes

Table 4. Comparison of chromatin accessibility and promoter methylation in the liver of cows from lowconcentrate (LC) and high-concentrate (HC) groups $^{1}$

\begin{tabular}{lccc}
\hline Item & LC & HC & Fold change $^{2}$ \\
\hline Chart (protection, \%) & & & \\
Area A (TSP) & & & \\
Area B (SRE) & $42 \pm 5$ & $64 \pm 6^{* *}$ & 1.77 \\
DNA-methylation & $36 \pm 3$ & & \\
Area A (TSP) & $0.12 \pm 0.03$ & $0.41 \pm 0.05^{* *}$ & 3.46 \\
Area B (SRE) & $0.10 \pm 0.03$ & $0.55 \pm 0.04^{* *}$ & 5.41 \\
\hline
\end{tabular}

${ }^{1}$ Values are means \pm SEM.

${ }^{2}$ Fold change of value in $\mathrm{HC}$ group compared with LC group.

${ }^{3} \mathrm{TSP}=$ transcription start position

${ }^{4} \mathrm{SRE}=$ sterol response elements.

$* * P<0.01$, as compared with LC group. 
accompanying their enhanced expression (Xu et al., 2016). These systemic effects of E. coli mastitis are conceivably mediated through LPS, just as during SARA. This paradox may indicate that systemically increased LPS levels require a longer duration before epigenetic mechanisms come into play for regulating the genes involved in lipid metabolism. The mastitis experiment was a short-term infection, lasting only for $24 \mathrm{~h}$. Treatment in the current study was much longer, and hence chromatin remodeling at the SCD1 promoter ensures imprinting of a lasting memory rather than activating acutely acting mechanisms. This is different from the acute function of epigenetic mechanisms operating to control inflammatory genes, which are controlled in the liver in part by TLR signaling-induced chromatin modifications during SARA (Foster et al., 2007; Chang et al., 2015d).

\section{SARA May Lead Fatty Acids Toward Catabolism in Cattle Livers}

We found decreased expression of lipid synthesisrelated genes (DGAT1 and -2, PLIN2) and transcription factors that activate them (SREBF1). This was complemented by increased expression of genes related to fatty acid oxidation (CPT1A and ACOX1). These data indicate enhanced utilization of fatty acyl-CoA in the liver rather than synthesis of triglycerides; however, we found no effect of SARA on hepatic TAG concentration. We reasoned that this may be explained by downregulation of MTTP and $A P O B$, which encode enzymes responsible for the transport of triglycerides and for assembly and export of TAG-rich VLDL from the bovine liver (Bernabucci et al., 2004). Similar results were also reported in our recent study on acute high-grain challenge-induced SARA, which potentially leads to TAG accumulation in the liver. Therefore, a reduction in the secretion of TAG-rich VLDL in HC cows may retard the disappearance of TAG, which, together with reduced synthesis of TG due to repression of lipid synthesis genes, may lead to no net change in concentration between the 2 experimental groups.

On the other hand, activating inflammatory and acute phase responses requires significant amounts of energy. Increased expression of inflammatory genes results in greater energy demands and, therefore, energy expenditure pathways are switched on to support the acute phase response in the liver (Straub, 2014). Indeed, we previously measured inflammation markers in peripheral blood, and expression of $I L 1 B, I L 6$, and TNFA were all significantly increased after prolonged feeding of high-concentrate diet. Furthermore, changes in blood biomarkers of liver function, including increases in aspartate aminotransferase, alanine aminotransferase, and lactate dehydrogenase and decreases in total protein and albumin, revealed deleterious effects of our treatment on liver function; all these data have been published recently (Guo et al., 2017). Hence, fatty acid catabolism (oxidation), rather than synthesis of TAG, may potentially be triggered by SARA-induced systemic inflammatory responses. Similar conclusions have been previously reached based on an entirely different set of proteins detected in a global proteomics survey of livers from goats suffering from SARA (Jiang et al., 2014).

Interestingly, we observed that expression of $A C A C A$ and $F A S N$-encoding genes is not apparently affected by SARA. The activities of these enzymes are key ratelimiting factors for the very first steps in the synthesis of long-chain fatty acids in the livers of nonruminants (Munday and Hemingway, 1999). In contrast, genes encoding enzymes operating further downstream in the lipid biosynthesis pathway (fatty acid modifications rather than de novo synthesis) were downregulated. Maintaining sufficient expression levels of $A C A C A$ and $F A S N$, as the most crucial factors of lipid biosynthesis, may ensure maintenance of a basal supply of fatty acids for the entire body in monogastric animals. Moreover, de novo lipogenic activity is widely regarded to be lower in ruminant livers than other tissues (Bergen and Mersmann, 2005). Therefore, it is reasonable that the expression levels of neither $A C A C A$ nor FASN were affected by SARA-induced metabolic changes with regards to their activity in the bovine liver. Furthermore, as observed in the current study, C14:0 concentration was altered by SARA, but its concentration was generally very low compared with those of 16:0 and 18:0 in peripheral plasma. Clearly, further research is warranted to better elucidate the link between fatty acid content and SCD activity during hepatic adaptation to nutrition in ruminants.

In conclusion, the physiology of SCD1 expression has long been considered to be regulated by hormones and PUFA. This study shows at the molecular level that epigenetic mechanisms may be constituents of the gearbox relaying the function of these physiological effectors to the genetic element controlling SCD1 expression. Subacute ruminal acidosis, the condition induced through extended feeding of high-concentrate diet, reduces the abundance of SREBP1, a key activator of SCD1 expression, in the liver of the dairy cow. Moreover, it elicits local chromatin remodeling at the SCD1 promoter to hinder SREBP1c binding. These changes cooperatively secure sustained downregulation of SCD1 expression in the liver of the dairy cow. 


\section{ACKNOWLEDGMENTS}

This study was financially supported by grants from the National Natural Science Foundation of China (Beijing; Grants 31672618 and 31172371), the National Basic Research Program of China (Beijing; Grant 2011CB100802), and the Priority Academic Program Development of Jiangsu Higher Education Institutions (Nanjing, China; PAPD).

\section{REFERENCES}

Abaker, J. A., T. L. Xu, D. Jin, G. J. Chang, K. Zhang, and X. Z. Shen. 2017. Lipopolysaccharide derived from the digestive tract provokes oxidative stress in the liver of dairy cows fed a high-grain diet. J. Dairy Sci. 100:666-678.

Arisqueta, L., M. Nunez-Garcia, J. Ogando, I. Garcia-Arcos, B. Ochoa, P. Aspichueta, O. Fresnedo, and Y. Rueda. 2013. Involvement of lipid droplets in hepatic responses to lipopolysaccharide treatment in mice. Biochim. Biophys. Acta 1831:1357-1367.

Bauman, D. E., and J. M. Griinari. 2001. Regulation and nutritional manipulation of milk fat: Low-fat milk syndrome. Livest. Prod. Sci. 70:15-29.

Bené, H., D. Lasky, and J. M. Ntambi. 2001. Cloning and characterization of the human stearoyl-CoA desaturase gene promoter: transcriptional activation by sterol regulatory element binding protein and repression by polyunsaturated fatty acids and cholesterol. Biochem. Biophys. Res. Commun. 284:1194-1198.

Bergen, W. G., and H. J. Mersmann. 2005. Comparative aspects of lipid metabolism: Impact on contemporary research and use of animal models. J. Nutr. 135:2499-2502.

Bernabucci, U., B. Ronchi, L. Basirico, D. Pirazzi, F. Rueca, N. Lacetera, and A. Nardone. 2004. Abundance of mRNA of apolipoprotein b100, apolipoprotein e, and microsomal triglyceride transfer protein in liver from periparturient dairy cows. J. Dairy Sci. 87:2881-2888.

Bernard, L., M. Bonnet, C. Leroux, K. J. Shingfield, and Y. Chilliard. 2009. Effect of sunflower-seed oil and linseed oil on tissue lipid metabolism, gene expression, and milk fatty acid secretion in Alpine goats fed maize silage-based diets. J. Dairy Sci. 92:6083-6094.

Boucher, J., A. Kleinridders, and C. R. Kahn. 2014. Insulin receptor signaling in normal and insulin-resistant states. Cold Spring Harb. Perspect. Biol. 6:a009191.

Cedar, H., and Y. Bergman. 2009. Linking DNA methylation and histone modification: Patterns and paradigms. Nat. Rev. Genet. 10:295-304.

Chang, G., T. Xu, B. Brand, W. Petzl, X. Shen, and H. M. Seyfert. 2015a. Three promoters with different tissue specificity and pathogen inducibility express the toll-like-receptor 2 (TLR2)-encoding gene in cattle. Vet. Immunol. Immunopathol. 167:57-63.

Chang, G., K. Zhang, T. Xu, D. Jin, J. Guo, S. Zhuang, and X. Shen. 2015b. Epigenetic mechanisms contribute to the expression of immune related genes in the livers of dairy cows fed a high concentrate diet. PLoS One 10:e0123942.

Chang, G., K. Zhang, T. Xu, D. Jin, H.-M. Seyfert, X. Shen, and S. Zhuang. 2015c. Feeding a high-grain diet reduces the percentage of LPS clearance and enhances immune gene expression in goat liver. BMC Vet. Res. 11:67.

Chang, G., S. Zhuang, H. M. Seyfert, K. Zhang, T. Xu, D. Jin, J. Guo, and X. Shen. 2015d. Hepatic TLR4 signaling is activated by LPS from digestive tract during SARA, and epigenetic mechanisms contribute to enforced TLR4 expression. Oncotarget 6:38578-38590.

Chen, C., Y. M. Shah, K. Morimura, K. W. Krausz, M. Miyazaki, T. A. Richardson, E. T. Morgan, J. M. Ntambi, J. R. Idle, and F. J. Gonzalez. 2008. Metabolomics reveals that hepatic stearoyl-CoA desaturase 1 downregulation exacerbates inflammation and acute colitis. Cell Metab. 7:135-147.
Chen, Y., M. Oba, and L. L. Guan. 2012. Variation of bacterial communities and expression of Toll-like receptor genes in the rumen of steers differing in susceptibility to subacute ruminal acidosis. Vet. Microbiol. 159:451-459.

Colman, E., W. B. Fokkink, M. Craninx, J. R. Newbold, B. De Baets, and V. Fievez. 2010. Effect of induction of subacute ruminal acidosis on milk fat profile and rumen parameters. J. Dairy Sci. 93:4759-4773.

de Faria Amormino, S. A., T. C. Arao, A. M. Saraiva, R. S. Gomez, W. O. Dutra, J. E. da Costa, J. de Fatima Correia Silva, and P. R. Moreira. 2013. Hypermethylation and low transcription of TLR2 gene in chronic periodontitis. Hum. Immunol. 74:1231-1236.

De Koster, J. D., and G. Opsomer. 2013. Insulin resistance in dairy cows. Vet. Clin. North Am. Food Anim. Pract. 29:299-322.

De Oliveira, N. F., D. C. Andia, A. C. Planello, S. Pasetto, M. R. Marques, F. H. Nociti Jr., S. R. Line, and A. P. De Souza. 2011. TLR2 and TLR4 gene promoter methylation status during chronic periodontitis. J. Clin. Periodontol. 38:975-983.

Dong, G., M. Qiu, C. Ao, J. Zhou, K. Erdene, W. Xi, Z. Zhang, and Y. Yang. 2014. Feeding a high-concentrate corn straw diet induced epigenetic alterations in the mammary tissue of dairy cows. PLoS One 9:e107659.

Drackley, J. K. 1999. ADSA Foundation Scholar Award. Biology of dairy cows during the transition period: The final frontier? J. Dairy Sci. 82:2259-2273.

Düvel, K., J. L. Yecies, S. Menon, P. Raman, A. I. Lipovsky, A. L. Souza, E. Triantafellow, Q. Ma, R. Gorski, S. Cleaver, M. G. Vander Heiden, J. P. MacKeigan, P. M. Finan, C. B. Clish, L. O. Murphy, and B. D. Manning. 2010. Activation of a metabolic gene regulatory network downstream of mTOR complex 1. Mol. Cell 39:171-183.

Folch, J., M. Lees, and G. H. Sloane Stanley. 1957. A simple method for the isolation and purification of total lipides from animal tissues. J. Biol. Chem. 226:497-509.

Foster, S. L., D. C. Hargreaves, and R. Medzhitov. 2007. Gene-specific control of inflammation by TLR-induced chromatin modifications. Nature 447:972-978.

Guo, J., G. Chang, K. Zhang, L. Xu, D. Jin, M. S. Bilal, and X. Shen. 2017. Rumen-derived lipopolysaccharide provoked inflammatory injury in the liver of dairy cows fed a high-concentrate diet. Oncotarget 8:46769-46780.

Guo, Y., X. Xu, Y. Zou, Z. Yang, S. Li, and Z. Cao. 2013. Changes in feed intake, nutrient digestion, plasma metabolites, and oxidative stress parameters in dairy cows with subacute ruminal acidosis and its regulation with pelleted beet pulp. J. Anim. Sci. Biotechnol. $4: 31$

Hayden, M. S., and S. Ghosh. 2011. NF-kappaB in immunobiology. Cell Res. 21:223-244

Jiang, X. Y., Y. D. Ni, S. K. Zhang, Y. S. Zhang, and X. Z. Shen. 2014. Identification of differentially expressed proteins in liver in response to subacute ruminal acidosis (SARA) induced by highconcentrate diet. Asian-Australas. J. Anim. Sci. 27:1181-1188.

Kleen, J. L., G. A. Hooijer, J. Rehage, and J. P. T. M. Noordhuizen. 2003. Subacute ruminal acidosis (SARA): A review. J. Vet. Med. A Physiol. Pathol. Clin. Med. 50:406-414.

Liu, X., M. S. Strable, and J. M. Ntambi. 2011. Stearoyl CoA desaturase 1: Role in cellular inflammation and stress. Adv. Nutr. $2: 15-22$.

Loor, J. J., A. A. Elolimy, and J. C. McCann. 2016. Dietary impacts on rumen microbiota in beef and dairy production. Anim. Front. $6: 22$.

Malodobra-Mazur, M., A. Dziewulska, K. Kozinski, P. Dobrzyn, K. Kolczynska, J. Janikiewicz, and A. Dobrzyn. 2014. Stearoyl-CoA desaturase regulates inflammatory gene expression by changing DNA methylation level in 3T3 adipocytes. Int. J. Biochem. Cell Biol. 55:40-50.

Maryam, J., M. E. Babar, Z. Bao, and A. Nadeem. 2016. A novel selection signature in stearoyl-coenzyme A desaturase (SCD) gene for enhanced milk fat content in Bubalus bubalis. Trop. Anim. Health Prod. 48:1343-1349. 
Moioli, B., G. Contarini, A. Avalli, G. Catillo, L. Orru, G. De Matteis, G. Masoero, and F. Napolitano. 2007. Short communication: Effect of stearoyl-coenzyme A desaturase polymorphism on fatty acid composition of milk. J. Dairy Sci. 90:3553-3558.

Munday, M. R., and C. J. Hemingway. 1999. The regulation of acetylCoA carboxylase-a potential target for the action of hypolipidemic agents. Adv. Enzyme Regul. 39:205-234.

Osborne, T. F. 2000. Sterol regulatory element-binding proteins (SREBPs): Key regulators of nutritional homeostasis and insulin action. J. Biol. Chem. 275:32379-32382.

Payne, E., and C. J. Masters. 1971. The invitro incorporation of 14Clabelled fatty acids into ovine liver and omental adipose tissue. Int. J. Biochem. 2:623-643.

Pfaffl, M. W. 2001. A new mathematical model for relative quantification in real-time RT-PCR. Nucleic Acids Res. 29:e45.

Plaizier, J. C., D. O. Krause, G. N. Gozho, and B. W. McBride. 2008. Subacute ruminal acidosis in dairy cows: the physiological causes, incidence and consequences. Vet. J. 176:21-31.

Pullen, D. L., J. S. Liesman, and R. S. Emery. 1990. A species comparison of liver slice synthesis and secretion of triacylglycerol from nonesterified fatty acids in media. J. Anim. Sci. 68:1395-1399.

Ren, L. P., X. Yu, G. Y. Song, P. Zhang, L. N. Sun, S. C. Chen, Z J. Hu, and X. M. Zhang. 2016. Impact of activating transcription factor 4 signaling on lipogenesis in HepG2 cells. Mol. Med. Rep. 14:1649-1658.

Rincon, G., A. Islas-Trejo, A. R. Castillo, D. E. Bauman, B. J. German, and J. F. Medrano. 2012. Polymorphisms in genes in the SREBP1 signalling pathway and SCD are associated with milk fatty acid composition in Holstein cattle. J. Dairy Res. 79:66-75.

Schwenk, R. W., W. Jonas, S. B. Ernst, A. Kammel, M. Jahnert, and A. Schurmann. 2013. Diet-dependent alterations of hepatic Scd1 expression are accompanied by differences in promoter methylation. Horm. Metab. Res. 45:786-794.

Smith, Z. D., and A. Meissner. 2013. DNA methylation: Roles in mammalian development. Nat. Rev. Genet. 14:204-220.

St John, L. C., D. K. Lunt, and S. B. Smith. 1991. Fatty acid elongation and desaturation enzyme activities of bovine liver and subcutaneous adipose tissue microsomes. J. Anim. Sci. 69:1064-1073.

Straub, R. H. 2014. Interaction of the endocrine system with inflammation: A function of energy and volume regulation. Arthritis Res. Ther. 16:203.

Tao, H., G. Chang, T. Xu, H. Zhao, K. Zhang, and X. Shen. 2015. Feeding a high concentrate diet down-regulates expression of ACACA, LPL and SCD and modifies milk composition in lactating goats. PLoS One 10:e0130525.
Vahmani, P., K. E. Glover, and A. H. Fredeen. 2014. Effects of pasture versus confinement and marine oil supplementation on the expression of genes involved in lipid metabolism in mammary, liver, and adipose tissues of lactating dairy cows. J. Dairy Sci. 97:4174-4183.

Vanselow, J., W. Yang, J. Herrmann, H. Zerbe, H. J. Schuberth, W. Petzl, W. Tomek, and H. M. Seyfert. 2006. DNA-remethylation around a STAT5-binding enhancer in the alphaS1-casein promoter is associated with abrupt shutdown of alphaS1-casein synthesis during acute mastitis. J. Mol. Endocrinol. 37:463-477.

Vaure, C., and Y. Liu. 2014. A comparative review of toll-like receptor 4 expression and functionality in different animal species. Front. Immunol. 5:316.

Vels, L., C. M. Rontved, M. Bjerring, and K. L. Ingvartsen. 2009. Cytokine and acute phase protein gene expression in repeated liver biopsies of dairy cows with a lipopolysaccharide-induced mastitis. J. Dairy Sci. 92:922-934.

Xu, T., F. C. Cardoso, A. Pineda, E. Trevisi, X. Shen, F. Rosa, J. S. Osorio, and J. J. Loor. 2017. Grain challenge affects systemic and hepatic molecular biomarkers of inflammation, stress, and metabolic responses to a greater extent in Holstein than Jersey cows. J. Dairy Sci. 100:9153-9162.

Xu, T., X. Shen, and H. M. Seyfert. 2016. Stearoyl-CoA desaturase 1 expression is downregulated in liver and udder during E. coli mastitis through enhanced expression of repressive C/EBP factors and reduced expression of the inducer SREBP1A. BMC Mol. Biol. 17:16.

Xu, T., H. Tao, G. Chang, K. Zhang, L. Xu, and X. Shen. 2015. Lipopolysaccharide derived from the rumen down-regulates stearoylCoA desaturase 1 expression and alters fatty acid composition in the liver of dairy cows fed a high-concentrate diet. BMC Vet. Res. 11:52.

Zebeli, Q., and B. N. Ametaj. 2009. Relationships between rumen lipopolysaccharide and mediators of inflammatory response with milk fat production and efficiency in dairy cows. J. Dairy Sci. 92:3800-3809.

Zebeli, Q., S. M. Dunn, and B. N. Ametaj. 2011. Perturbations of plasma metabolites correlated with the rise of rumen endotoxin in dairy cows fed diets rich in easily degradable carbohydrates. J. Dairy Sci. 94:2374-2382.

Zhang, K., G. Chang, T. Xu, L. Xu, J. Guo, D. Jin, and X. Shen 2016. Lipopolysaccharide derived from the digestive tract activates inflammatory gene expression and inhibits casein synthesis in the mammary glands of lactating dairy cows. Oncotarget 7:9652-9665. 